\title{
Blefaroplastia inferior: poderia a cirurgia proporcionar satisfação aos pacientes?
}

\section{Lower blepharoplasty: would the surgery provide satisfaction to the patient?}

Giovanni André Pires Viana ${ }^{1}$, Midori Hentona OsakI ${ }^{2}$, Mauro Nishi ${ }^{3}$

\section{RESUMO}

Objetivo: Foi realizado um estudo prospectivo com objetivo de avaliar os resultados de cinquenta pacientes submetidos a blefaroplastia inferior transcutânea, visando a análise do resultado clínico e a satisfação dos pacientes na Universidade Federal de São Paulo, entre abril de 2005 e maio de 2007

Métodos: Os pacientes foram alocados aleatoriamente em dois grupos cirúgicos. O Grupo Cirúrgico 1 (Grupo Controle) foi composto por 25 pacientes submetidos a blefaroplastia inferior tradicional e cantopexia lateral de rotina. O Grupo Cirúrgico 2 (Grupo Experimental) foi composto por 25 pacientes submetidos a blefaroplastia inferior com transposição das bolsas adiposas e cantopexia lateral de rotina. Para avaliar os resultados obtidos foi utilizado a avaliação da autoestima dos pacientes, por meio da Escala de Autoestima de Rosenberg UNIFESP/EPM. O outro método utilizad o foi solicitar a participação de três cirurgiões independentes que avaliaram as fotografias de pré e pós-operatório e com auxílio de uma escala topográfica, quantificaram os resultados.

Resultados: A média de idade foi de 48,8 anos, com predomínio do sexo feminino (96\%). A análise das fotografias mostrou que $96 \%$ dos pacientes apresentaram meIhora significativa. A autoestima melhorou de um escore médio no pré-operatório de 5,1 (desvio padrão $=4,1$ ) para um valor médio de 3,6 (desvio padrão $=3,5$ ) após 6 meses da cirurgia $(p=0,001)$.

Conclusão: Os autores concluíram que ambos os procedimentos seriam segurose eficazes, com baixo índice de complicação, apresentando melhora da autoestima, visível após seis meses da cirurgia.

Descritores: Pálpebras/cirurgia; Blefaroplastia; Autoimagem; Estética; Autoestima; Satisfação do paciente; Questionários

\begin{abstract}
Purpose: The purpose of this study was to analyze prospectively fifty patients submitted to lower eyelid blepharoplasty at the Federal University of São Paulo, between April 2005 and May 2007.

Methods: Fifty patients were assigned to interventions into two surgical groups by using random allocation. The Surgical Group 1 (control group) was composed of 25 patients who were submitted to conservatively standard fat-resection lower eyelid blepharoplasty, and routine lateral canthal support. The Surgical Group 2 (experimental group) was represented by 25 patients submitted to lower eyelid blepharoplasty with periorbital fat mobilization and arcus marginalis redrape, and routine lateral canthal support. The self-esteem of all patients was compared with those in 25 age-matched volunteers from the general population. The parameters of the Rosenberg Self-Esteem Scale were determined preoperatively and at 6-month interval postoperatively. Standardized photographs obtained before and after surgery were evaluated by three independent observers. Results: The median follow-up was 395 days (range 364 to 547 days). The mean age was 48.8 years, the population's gender was predominantly female (96\%). Analysis of preoperative and postoperative photographs showed that $96 \%$ patients achieved significantimprovement. Self-esteem scores improved from baseline preoperative mean levels of 5.1 (Standard Deviation = 4.1) to a mean level of 3.6 (Standard Deviation =3.5) at 6 months post-surgery $(p=0.001)$. No patients had orbital hematoma, blepharitis, lagophthalmos or ectropion.
\end{abstract}

Conclusions: The authors concluded that both procedures are safe and effective with low complication rates, and marked improvement in self-esteem was observed in patients at 6-month follow-up.

Keywords: Eyelids/surgery; Blepharoplasty; Self concept; Esthetics; Patient satisfaction; Questionnaires

\section{INTRODUÇÃO}

O processo de envelhecimento na região periorbital pode causar inúmeras mudanças, entre as quais poder-se-ia citar as alterações na qualidade ou quantidade de pele, a herniação das bolsas adiposas ou o alongamento da margem palpebral inferior. As queixas comuns incluiriam as bolsas adiposas, linhas de expressão ou olhar cansado. Os avanços recentes sobre a compreensão da topografia dos compartimentos adiposos da face, a perda de volume dos tecidos da face durante o envelhecimento e a descrição detalhada dos ligamentos faciais tem propiciado o melhoramento das técnicas de rejuvenescimento facial e periorbital ${ }^{(1-4)}$. O tratamento cirúrgico da pálpebra inferior através da incisão transcutânea, tradicionalmente tem envolvido a escolha ou de um retalho cutâneo ou de um retalho músculo-cutâneo, havendo pouca diferença de resultados entre os dois procedimentos ${ }^{(5-10)}$.
Atualmente, existem duas vertentes quando se aborda o tema sobre blefaroplastia inferior, uma que advoga o uso de técnica cirúrgica mais agressiva, maximizando o resultado enquanto que a outra, mais conservadora, teria como objetivo minimizar o risco de complicações. Loeb(11) foi um dos primeiros cirurgiões a preservar o tecido adiposo durante a blefaroplastia inferior, entretanto desde que de la Plaza e Arroyo ${ }^{(12)}$ descreveram sobre o reparo das bolsas adiposas durante a blefaroplastia inferior, o interesse pela abordagem conservadora e a sua preservação tem gerado grande interesse.

A avaliação dos resultados em cirurgia plástica é especialmente pertinente, pois a satisfação do paciente é o fator preponderante na determinação do sucesso. Normalmente, esta avaliação se baseia em comparações subjetivas de imagens fotográficas selecionadas, entretanto deveria ser considerada de baixa confiabilidade. A avaliação do aspecto psicológico do paciente e suas expectativas em relação
Financiamento: Não houve financiamento para este trabalho.

Divulgação de potenciais conflitos de interesse: G.A.P.Viana, Nenhum; M.H.Osaki, Nenhum; M.Nishi, Nenhum.

Endereço para correspondência: Giovanni André Pires Viana. Av. Lavandisca , 741 - Conj. 53 - São Paulo (SP) - 04515-011 - Brasil - E-mail: info@cliniplast.com

Registrado no Australian New Zealand Clinical Trials Registry - ACTRN12609000732280 (http:// www.actr.org.au) 
à cirurgia também deveriam ser analisadas. Para tanto, uma avaliação mais objetiva dos resultados poderia fornecer orientação mais confiável sobre o padrão preferencial na prática clínica do dia-a-dia. Deste modo, diferentes escalas de mensuração estão sendo adotadas em diferentes situações, para comparar os resultados cirúrgicos ${ }^{(13-15)}$.

O objetivo deste estudo foi avaliar o resultado cirúrgico e a satisfação de 50 pacientes submetidos a blefaroplastia inferior transcutânea, na Universidade Federal de São Paulo, no setor de Cirurgia Plástica Ocular.

\section{MÉTODOS}

Realizou-se um estudo prospectivo, randomizado e controlado entre abril de 2005 e maio de 2007 na Universidade Federal de São Paulo, no setor de Cirurgia Plástica Ocular.

\section{AMOSTRA DA POPULAÇÃo}

Cinquenta pacientes consecutivos foram recrutados no ambulatório e todos que concordaram em participar da pesquisa assinaram o termo de consentimento, aprovado pelo Comitê de Ética em Pesquisa da UNIFESP. Os participantes tinham idade entre 30 e 65 anos.

A avaliação pré-operatória incluiu exame oftalmológico e exames específicos referentes a qualquer condição médica relevante. Pacientes com história de lesão ou cirurgia prévia na pálpebra inferior foram excluídos do estudo.

\section{Cirurgia e seguimento}

Todos os 50 participantes foram submetidos a blefaroplastia inferior transcutânea, sendo operados pelo mesmo cirurgião (GAPV). Todos foram alocados em dois grupos cirúrgicos de modo aleatório (através de sorteio - "lottery draw"), sendo que cada grupo foi composto por 25 pacientes.

A cirurgia foi realizada sob anestesia local, através do bloqueio do nervo infraorbital com lidocaína a 2\% a 1:200.000. No Grupo Cirúrgico 1 (GC1) foi realizado a blefaroplastia inferior tradicional, com retirada cautelosa do excesso das bolsas adiposas. No Grupo Cirúrgico 2 (GC2) realizou-se a blefaroplastia inferior, com tratamento das bolsas adiposas conservadoramente, com posterior transposição das bolsas medial e média, sendo suturadas ao periósteo com fio de poliglactina 910, número 6-0.

Todos os pacientes receberam alta hospitalar no mesmo dia. O primeiro retorno foi em torno do quinto dia, sendo realizado a verificação da ferida cirúrgica e qualquer complicação. O segundo e o terceiro retornos foram planejados para o 1ํ e 3으 mês após a cirurgia. O quarto retorno foi planejado para o 60 mês. O último acompanhamento foi agendado para quando completasse um ano da cirurgia.

\section{Avaliação da FLACidez PalPebral}

A flacidez foi avaliada em todos os pacientes através da avaliação do tônus da margem palpebral por meio do teste de distração anterior ${ }^{(16-20)}$. A medida foi realizada no trans-operatório com ajuda de um compasso de Castroviejo, anotando-se os valores obtidos antes e depois da cantopexia. Adotou-se o valor maior que $12 \mathrm{~mm}$ como indicativo de frouxidão da pálpebra inferior.

Outro método de se avaliar a flacidez palpebral foi através do pinçamento da face lateral da pálpebra inferior e tracionando-a lateralmente no sentido horizontal, tendo-se o cuidado de se manter a posição da margem palpebral tangenciando o limbo inferior (teste de distração lateral). A flacidez foi definida como a distância lateral do deslocamento da comissura lateral sobre o ponto onde a margem palpebral sobrepor-se-ia a margem do rebordo lateral da órbita, sendo considerado neste estudo, o valor maior que $7 \mathrm{~mm}$.

Um fio de náilon número 5-0 foi usado para a realização da cantopexia. O exato local da colocação desta sutura foi variável, dependendo do resultado da exoftalmometria e da inclinação cantal preexistente ("Canthal tilt"). A exoftalmometria foi realizada no préoperatório, com auxílio do exoftalmomêtro de Luedde, pelo mesmo examinador em todos os casos e repetido duas vezes para cada paciente, adotando-se a média para cada resultado.

\section{Tratamento do excesso de PELE}

A lamela anterior foi tracionada em um vetor supero-lateral, ao invés de um vetor vertical puro. A excisão do excesso de pele foi feita através da remoção de um triângulo de tecido lateralmente ao canto, minimizando assim a quantidade de tecido removido. Concomitantemente a cantopexia, realizou-se a suspensão do músculo orbicular, ou seja, foi realizada a sutura da porção pré-septal do músculo orbicular ao periósteo do rebordo lateral da órbita com um ponto simples (Poliglactina 910, número 6-0). A síntese da lesão foi realizada livre de tensão com náilon 6-0.

\section{ANÁlise dos RESULtAdos}

Para conseguir algum nível de quantificação dos resultados, uma escala topográfica foi utilizada para avaliar os resultados pré e pósoperatório (0 - melhor resultado; 3 - pior resultado) ${ }^{(17)}$. Cada paciente foi submetido a avaliação fotográfica no pré e pós-operatório em cada retorno. As fotografias foram realizadas por máquina digital Olympus Stylus 710, as sequências (plano frontal olhos abertos e fechados, perfil direito e esquerdo) de cada paciente foram realizadas em um tempo único, portanto sob as mesmas condições de iluminação (natural e com flash). As fotografias de antes e depois foram analisadas por três cirurgiões que não estiveram envolvidos com os pacientes. Os dados fornecidos pelos cirurgiões foram agrupados e as médias foram utilizadas em todas as comparações.

\section{QuestionáRIo UTILIZAdo No ESTUdo}

Para avaliar e quantificar o resultado da cirurgia, todos os pacientes foram submetidos à avaliação da autoestima através da Escala de Autoestima de Rosenberg. Esta escala foi traduzida e validada a língua portuguesa por Dini et al.(18), sendo denominada Escala de Autoestima de Rosenberg UNIFESP/EPM (RSES-EPM). A escala é composta por 10 perguntas, cada qual com quatro alternativas. Cada pergunta poderá variar entre zero (concordo plenamente) e três (discordo plenamente). O escore total da escala variará entre zero e 30 pontos, sendo que quanto menor o escore, melhor será a autoestima.

Para melhor análise dos resultados, criou-se um grupo controle de autoestima (GCon), composto por 25 funcionários da instituição, que não desejava ser submetido a nenhum procedimento cirúrgico (cirurgia plástica) no período de pelo menos 6 meses.

Os participantes do Grupo Cirúrgico responderam a RSES-EPM no pré-operatório e no $6^{\circ}$ mês após a cirurgia, enquanto que os voluntários do Grupo Controle foram avaliados em dois momentos distintos, com intervalo de 6 meses entre as duas avaliações.

\section{Análise estatística}

Foi utilizado o teste de Wilcoxon para comparar os resultados do questionário de autoestima nos dois momentos distintos. $\mathrm{O}$ teste de Mann-Whitney foi utilizado para analisar a importância da avaliação subjetiva das fotografias. As diferenças seriam consideradas significativas se a probabilidade fosse inferior a 0,05.

\section{RESULTADOS}

\section{Cirurgia e seguimento}

A tabela 1 demonstra as características sociodemográficas de ambos os grupos cirúrgicos. A idade média da população foi de 48,8 anos (34 - 65); houve predominância do sexo feminino (96\%). Não houve diferença no tempo cirúrgico entre os dois grupos; o período de acompanhamento foi de pelo menos 1 ano (364 - 547 dias). 
O resultado da exoftalmometria realizado em todos os pacientes pode ser observado na tabela 2. A frequência de esclera aparente no pré-operatório foi mais comum à medida que os valores obtidos na exoftalmometria aumentaram. Pacientes com exoftalmometria $\geq 20 \mathrm{~mm}$ tiveram $28,5 \%$ de incidência de esclera aparente no préoperatório.

Não houve diferença estatística entre os grupos em relação à flacidez palpebral, entretanto a cantopexia se mostrou eficaz em reduzir a flacidez antes e depois da cirurgia, conforme demonstrado pela tabela 3.

Os resultados da análise pelos três observadores independentes através de uma escala proporcional topográficas são vistos na tabela 4.

\section{QuestionáRIO UTILIZAdo NO ESTUdo}

A análise descritiva mostrou que a média da avaliação da autoestima no pré-operatório foi de 5,1 ( $D P=4,1)$, enquanto que no pósoperatório foi de 3,6 ( $D P=3,5)$. A mediana no primeiro momento foi de 4,5 e após 6 meses foi de 3,0. O intervalo de confiança (95\%) para o questionário no pré-operatório foi de 3,$99 ; 6,21$, enquanto que no período pós-operatório foi de 2,$64 ; 4,92$.

A análise da RSES-EPM mostrou que 31 (62\%) indivíduos tiveram melhora da autoestima após a cirurgia, 12 (24\%) pacientes tiveram sua pontuação inalterada e em 7 (14\%) casos houve piora. Em média, houve uma redução de 1,5 na pontuação pós-operatória (Figura 1).

Tabela 1. Aspectos sociodemográficos

\begin{tabular}{|c|c|c|c|}
\hline \multirow[b]{2}{*}{ Variável } & \multicolumn{2}{|c|}{ Grupo cirúrgico } & \multirow[b]{2}{*}{ Valor de $\mathrm{p}$} \\
\hline & $\mathrm{GC1}(\mathrm{N}=25)$ & GC2 (N=25) & \\
\hline \multicolumn{4}{|l|}{ Idade (anos) } \\
\hline Média (DP) & $49,5(6,6)$ & $48,1(5,9)$ & \multirow{2}{*}{0,433} \\
\hline Mínimo - máximo & $34-65$ & $40-65$ & \\
\hline \multicolumn{4}{|l|}{ Faixa etária - N(\%) } \\
\hline $30-39$ anos & $2(8,0 \%)$ & $0(0,0 \%)$ & \multirow{4}{*}{0,074} \\
\hline $40-49$ anos & $10(40,0 \%)$ & $18(72,0 \%)$ & \\
\hline $50-59$ anos & $11(44,0 \%)$ & $6(24,0 \%)$ & \\
\hline$\geq 60$ anos & $2(8,0 \%)$ & $1(4,0 \%)$ & \\
\hline \multicolumn{4}{|l|}{ Sexo - N(\%) } \\
\hline Feminino & $24(96,0 \%)$ & $24(96,0 \%)$ & \multirow{2}{*}{1,000} \\
\hline Masculino & $1(4,0 \%)$ & $1(4,0 \%)$ & \\
\hline \multicolumn{4}{|l|}{ Duração cirurgia (horas) } \\
\hline Média (DP) & $1,47(0,51)$ & $1,57(0,41)$ & \multirow{3}{*}{0,078} \\
\hline Mediana & 1,25 & 1,50 & \\
\hline Mínimo - máximo & $1,0-3,0$ & $1,1-3,0$ & \\
\hline
\end{tabular}

Tabela 2. Exoftalmometria

\begin{tabular}{lccccc}
\hline $\begin{array}{l}\text { Medida da } \\
\text { exoftalmometria } \\
\text { (milímetros) }\end{array}$ & \multicolumn{2}{c}{ GC 1 } & & \multicolumn{2}{c}{ GC 2 } \\
\cline { 2 - 3 } \cline { 5 - 6 } \cline { 5 - 6 } Média (DP) & OD & OE & & OD & OE \\
\hline Mediana & $16,04(2,74)$ & $16,84(2,49)$ & & $15,72(2,59)$ & $17,08(2,03)$ \\
Mínimo - máximo & 15 & 17 & & 16 & 16 \\
IC (95\%) & $12-23$ & $14-22$ & & $10-21$ & $13-22$ \\
\hline
\end{tabular}

OD: olho direito; OE: olho esquerdo; DP: desvio padrão; GC1: cirurgia tradicional; GC2: cirurgia com transposição de bolsas; IC: intervalo de confiança.

\section{Complicações}

Cinco pacientes apresentaram mau posicionamento da pálpebra inferior após um ano de seguimento, caracterizado pela esclera aparente (lateralmente), sendo que quatro destes pacientes tinham exoftalmometria com valor $\geq 18 \mathrm{~mm}$. Seis pacientes apresentavam esclera aparente no pré-operatório, mas nenhum deles desejou alteração no posicionamento da pálpebra inferior.

Dois pacientes apresentaram quemose, necessitando usar colirío de fluormetolona a 0,1\% (1 gota em cada olho, 4 vezes ao dia, por 7 dias), com resolução completa. Nenhum paciente apresentou hematoma orbital, blefarite, lagoftalmo ou ectrópio da pálpebra inferior.

\section{DISCUSSÃO}

A evolução da blefaroplastia inferior resultou em conceitos divergentes, onde alguns autores indicariam a preservação das bolsas adiposas da pálpebra inferior e ressecção mínima da pele, outros evitariam lesar o músculo orbicular dos olhos, enquanto alguns recomendariam o uso de retalho musculocutâneo com amplo desco-

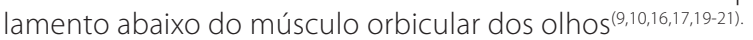

A etiopatogenia do processo de envelhecimento periorbital é multifatorial. As mudanças nesta região relacionadas à idade incluiriam o aparecimento de ritides, esclera aparente, deflação da região infraorbital, protusão das bolsas adiposas, excesso de pele na pálpebra superior e inferior, festões, entre outros. Além disso, a atenuação

Tabela 3. Teste distração

\begin{tabular}{|c|c|c|c|c|}
\hline \multirow[b]{2}{*}{$\begin{array}{l}\text { Teste } \\
(\mathrm{N}=50)\end{array}$} & \multicolumn{2}{|c|}{ Avaliação } & \multirow[b]{2}{*}{ Variação } & \multirow[b]{2}{*}{$\begin{array}{l}\text { Valor } \\
\text { de p }\end{array}$} \\
\hline & $\begin{array}{c}\text { Antes da } \\
\text { cantopexia }\end{array}$ & $\begin{array}{l}\text { Depois da } \\
\text { cantopexia }\end{array}$ & & \\
\hline \multicolumn{5}{|l|}{ TDA OD } \\
\hline Média (DP) & $5,5(1,5)$ & $4,1(1,0)$ & $1,4(0,8)$ & \\
\hline Mediana & 5 & 4 & 1 & $<0,001$ \\
\hline Mínimo - máximo & $3-11$ & $2-7$ & $0-4$ & \\
\hline \multicolumn{5}{|l|}{ TDA OE } \\
\hline Média (DP) & $5,2(1,7)$ & $3,9(1,3)$ & $1,2(1,0)$ & \\
\hline Mediana & 5 & 4 & 1 & $<0,001$ \\
\hline Mínimo - máximo & $2-12$ & $1-7$ & $-1-5$ & \\
\hline \multicolumn{5}{|l|}{ TDL OD } \\
\hline Média (DP) & $3,4(1,2)$ & $2,4(0,8)$ & $1,0(0,6)$ & \\
\hline Mediana & 3 & 2 & 1 & $<0,001$ \\
\hline Mínimo - máximo & $2-7$ & $1-5$ & $0-3$ & \\
\hline \multicolumn{5}{|l|}{ TDL OE } \\
\hline Média (DP) & $3,2(1,2)$ & $2,2(0,6)$ & $1,0(0,8)$ & \\
\hline Mediana & 3 & 2 & 1 & $<0,001$ \\
\hline Mínimo - máximo & $2-7$ & $1-5$ & $0-4$ & \\
\hline
\end{tabular}

TDA: teste distração anterior; TDL: teste distração lateral; OD: olho direito; OE: olho esquerdo; DP: desvio padrão.

Tabela 4. Análise das fotografias no pré e pós-operatório

\begin{tabular}{lcc}
\hline & \multicolumn{2}{c}{ Análise } \\
\cline { 2 - 3 } & Pré & Pós \\
\hline Mínimo - máximo & $0,6-2,3$ & $0,3-1,6$ \\
Intervalo de confiança (95\%) & 1,$42 ; 1,66$ & 0,$77 ; 0,96$ \\
\hline
\end{tabular}




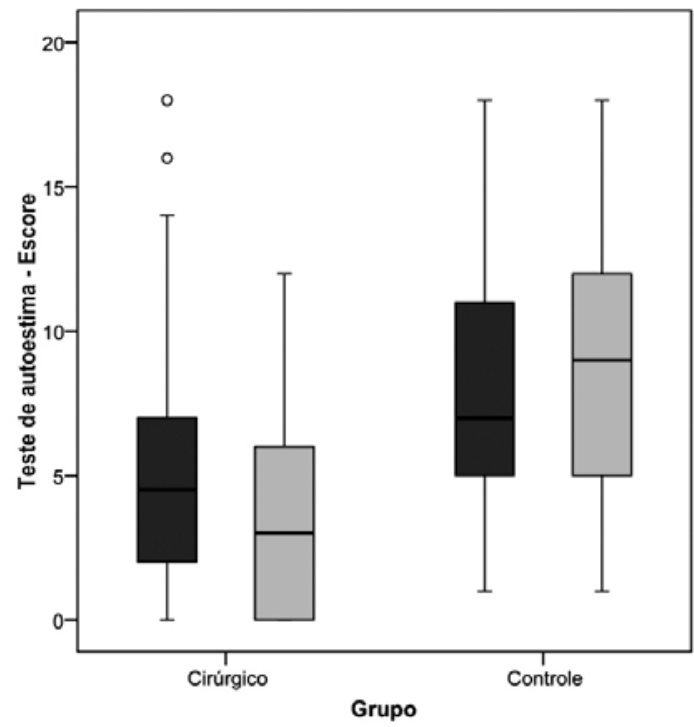

Avaliação $\square$ Antes $\square$ Depois

Figura 1. Box-plot demonstrando a autoestima em dois momentos distintos no grupo cirúrgico $\mathrm{X}$ grupo controle.

do tendão cantal lateral resultaria em perda da arquitetura jovial dos olhos, secundária a uma diminuição da inclinação superior do canto lateral que seria esteticamente agradável| $\left.\right|^{(4,21-23)}$. A mudança na posição do canto lateral é funcionalmente importante, pois sua alteração contribuiria para a flacidez da pálpebra inferior, o que poderia resultar no arredondamento da comissura lateral e estreitamento da fenda palpebral| ${ }^{(16,19,20)}$.

Historicamente, a complicação mais comum após a blefaroplastia inferior é o mau posicionamento palpebral, com incidência variando entre $5 \%$ a $90 \%{ }^{(16,24)}$. O fator etiológico mais prevalente no mau posicionamento da pálpebra inferior é a deficiência vertical da lamela anterior ou posterior, associada a frouxidão tarsoligamentar ${ }^{(16,24)}$. Para evitar a deformidade típica vista após este procedimento, a cantopexia ou a cantoplastia tem sido adotadas como método de suporte de rotina durante a blefaroplastia inferior $r^{(16,19,20,25)}$.

A avaliação da posição ântero-posterior do globo ocular em relação à órbita (exoftalmometria) é importante porque torna possível a identificação de pacientes com olhos proeminentes e morfologia de vetor negativo. Estes pacientes estariam em maior risco de mau posicionamento da pálpebra inferior, necessitando de suporte adicional no canto lateral. Neste estudo, o resultado da exoftalmometria em pacientes submetidos a blefaroplastia inferior foi analisado e os autores documentaram os parâmetros pré-operatórios relativos a suas características periorbitais. A primeira constatação foi que 50\% dos pacientes tiveram como resultado o valor entre 15-17 mm, e apenas $4 \%$ destes pacientes tinham esclera aparente no pré-operatório, ao contrário do resultado demonstrado por alguns autores ${ }^{(23)}$. Um outro achado interessante foi que 15,8\% dos pacientes com olhos proeminentes ( $\geq 18 \mathrm{~mm}$ ) tinham um vetor neutro durante o exame clínico, mas sendo identificados durante a exoftalmometria.

A cantopexia foi associada para evitar o mau posicionamento da pálpebra inferior que é a complicação mais comum após este tipo de cirurgia. Não houve diferença significativa na flacidez da pálpebra inferior entre o dois grupos estudados, no entanto, a cantopexia teve um grande impacto na flacidez da pálpebra inferior, como demonstrado por vários autores ${ }^{(16,19,20,23)}$. Em geral, todos os métodos de cantopexia/cantoplastia corrigem a frouxidão tarsoligamentar, contrapondo-se às forças de cicatrização ${ }^{(16,19,20,23)}$.

Embora houvesse casos com flacidez importante tanto no teste de distração anterior quanto no teste de distração lateral e sendo esta flacidez indicativa de se realizar a cantoplastia, os autores optaram por usar a cantopexia e analisar a evolução desses pacientes ${ }^{(5,23)}$. Durante a análise dos resultados pré e pós-operatório da cantopexia, viu-se que a frouxidão tarsoligamentar melhorou em todos os casos, com exceção de cinco pacientes que apresentaram esclera aparente. Os autores observaram que, apesar de muitos estudos que avaliaram a frouxidão tarsoligamentar da pálpebra inferior e seu tratamento através da cantopexia ou cantoplastia, não houve nenhum estudo quantificando estes dados no pré e pós-operatório, demonstrando numericamente a melhora após o tratamento cirúrgico.

Os autores compararam os resultados cirúrgicos entre dois grupos de pacientes submetidos a blefaroplastia inferior tradicional e a blefaroplastia inferior com mobilização das bolsas adiposas e mobilização do arco marginal. Quando se opta por realizar a blefaroplastia com preservação e mobilização das bolsas adiposas, presume-se que o volume destas bolsas não estejam aumentados, sendo sua preservação importante para a manutenção da jovialidade da projeção do globo ocular em relação a face ${ }^{(8,9,16,19)}$. Quando os autores compararam o tempo de cirurgia entre os dois grupos, observou-se que não houve diferença estatisticamente significante entre eles ${ }^{(26)}$.

Durante a análise das fotografias observou-se que a maioria dos pacientes tinha flacidez cutânea (74\%), bolsas adiposas na pálpebra inferior (76\%) e deformidade denominada de sulco nasojugal pronunciado ("tear trough") (74\%). Estas três condições foram as principais responsáveis pelo procura de tratamento cirúrgico.

Os objetivos da cirurgia plástica seriam remodelar as estruturas normais e restaurar a aparência jovial, melhorando além da aparência a autoimagem do paciente. Um número crescente de estudos tem relatado que a motivação para a cirurgia plástica não poderia ser explicada exclusivamente por uma simples relação de causalidade entre a personalidade e a deformidade. Eles tem salientado a importância dos aspectos interpessoais e sociais ${ }^{(13-15,18,27)}$. Tradicionalmente, o método de avaliação de resultado mais utilizado em cirurgia plástica tem sido baseado na comparação de fotografias de pré e pós-operatório. Outra possibilidade utilizada pelos cirurgiões seria analisar a incidência de complicação em cada intervenção. Infelizmente, nenhum desses métodos tem se mostrado útil na avaliação de resultado, pois eles não seriam confiáveis e nem validados, ou seja, não seguiriam metodologia adequada e reprodutível.

Os autores compararam os resultados cirúrgicos da blefaroplastia inferior transcutânea não com base na análise subjetiva das fotografias de pré e pós-operatório, mas através de dois modos, o primeiro com base na avaliação da autoestima dos pacientes e o segundo baseado na avaliação independente de três cirurgiões convidados, que não estiveram envolvidos com esses pacientes, para avaliar as fotografias (pré e pós-operatório) por meio de uma escala topográfica ${ }^{(17)}$.

Observou-se que a maioria dos pacientes (96\%) teve melhora da pálpebra inferior após a cirurgia na avaliação independente. A análise independente demonstrou um resultado final global de 0,84 em uma escala de 0 - 3 (sendo "0" o melhor resultado possível), que os autores consideraram ser um nível aceitável de melhora

O resultado global da RSES-EPM no período pré-operatório foi em média 5,1 enquanto que no pós-operatório foi de 3,6. Apesar de todos os participantes terem relatado mudanças positivas em suas vidas sociais e relações interpessoais, observou-se que 7 (14\%) pacientes apresentaram piora da autoestima. Entre estes pacientes, os autores procuraram uma explicação para este evento (piora) e encontraram algumas situações especiais: três pacientes se divorciaram, três tiveram problemas com seus filhos e uma ficou viúva após a cirurgia. Figueroa mostrou que a dor e a perda seriam responsáveis pela rutura da imagem corporal com alteração significativa da autoestima, o que poderia durar até um ano após o evento(28).

Observou-se neste trabalho melhora na autoestima após a cirurgia, demostrando que a desaprovação com o corpo estaria diretamente relacionada à baixa autoestima, como demonstrado por diversos autores ${ }^{(28,29)}$. Estes resultados tem apontado que atualmente, 
a sociedade e o mercado de trabalho tem exigido uma aparência cada vez mais jovial, mostrando que desde a primeira vez que Narciso viu o reflexo de seu rosto em um lago, a humanidade está obcecada com a sua aparência.

No entanto, algumas limitações estão presentes neste estudo, pois mesmo sendo o instrumento de mensuração psicométrico, continua sendo difícil estabelecer relações causais entre as variáveis. Aspectos importantes de disfunção física e psicológica podem ter sido perdidos pelo questionário usado. A variável independente (intervenção cirúrgica) não poderia ser manipulada por causa das restrições éticas e práticas. Portanto, um projeto prospectivo de pesquisa, onde os próprios participantes serviriam como controle, foi a opção mais viável e adequada para testar as hipóteses do estudo. No futuro, será necessário considerar como as medidas psicométricas adicionais da imagem corporal irão permitir o aperfeiçoamento da compreensão dos resultados da qualidade de vida e da autoestima rotineiramente vivenciados pelos pacientes de cirurgia plástica.

\section{CONCLUSÃO}

Esse estudo demonstrou que ambas as técnicas cirúrgicas apresentaram bons resultados, com baixa incidência de complicações. Os resultados desta pesquisa confirmaram a hipótese que a blefaroplastia inferior melhoraria a aparência física, produzindo um efeito psicológico positivo através da melhora da autoestima, estando esta melhora visível no 6ํㅡês após a cirurgia.

\section{AGRADECIMENTOS}

Os autores gostariam de agradecer aos três cirurgiões independentes: Angelino Júlio Cariello, Daniel Nunes e Silva e Renato Wendell Damasceno.

\section{REFERÊNCIAS}

1. Rohrich RJ, Pessa JE. The fat compartments of the face: anatomy and clinical implications for cosmetic surgery. Plast Reconstr Surg. 2007;119(7):2219-27. Comment in: Plast Reconstr Surg. 2008;121(3):1061; author reply 1061-2.

2. Rohrich RJ, Arbique GM, Wong C, Brown S, Pessa JE. The anatomy of suborbicularis fat: implications for periorbital rejuvenation. Plast Reconstr Surg. 2009;124(3):946-51.

3. Lambros V. Observations on periorbital and midface aging. Plast Reconstr Surg. 2007; 120(5):1367-76; discussion 1377

4. Ghavami A, Pessa JE, Janis J, Khosla R, Reece EM, Rohrich RJ. The orbicularis retaining ligament of the medial orbit: closing the circle. Plast Reconstr Surg. 2008;121(3):994-1001.

5. Trussler AP, Rohrich RJ. MOC-PSSM CME article: Blepharoplasty. Plast Reconstr Surg. 2008;121(1 Suppl):1-10.

6. Spira M. Lower blepharoplasty: a clinical study. Plast Reconstr Surg. 1977;59(1):35-8.

7. Rizk SS, Matarasso A. Lower eyelid blepharoplasty: analysis of indications and the treatment of 100 patients. Plast Reconstr Surg. 2003;111(3):1299-306; discussion 1307-8.

8. Grant JR, LaFerriere KA. Periocular rejuvenation; lower eyelid blepharoplasty with repositioning and the suborbicularis oculi fat. Facial Plast Surg Clin North Am. 2010; 18(3):399-409.

9. Ben Simon GJ, McCann JD. Cosmetic eyelid and facial surgery. Surv Ophthalmol. 2008; 53(5):426-42.
10. Rohrich RJ, Ghavami A, Mojallal A. The five-step lower blepharoplasty: blending the eyelid-cheek junction. Plast Reconstr Surg. 2011;128(3):775-83. Comment in: Plast Reconstr Surg. 2012;129(5):841e-2e; author reply 842e-3e.

11. Loeb R. Fat pad sliding and fat grafting for leveling lid depressions. Clin Plast Surg. 1981;8(4):757-6.

12. De la Plaza R, Arroyo JM. A new technique for the treatment of palpebral bags. Plast Reconstr Surg. 1988;81(5):677-85.

13. Harris DL, Carr AT. The Derriford Appearance Scale (DAS59): a new psychometric scale for the evaluation of patients with disfigurements and aesthetic problems of appearance. Br J Plast Surg. 2001;54(3):216-22

14. Jenkinson C, Coulter A, Wright L. Short-form 36 (SF36) health survey questionnaire: normative data for adults of working age. BMJ. 1993:306(6890):1437-40. Comment in: BMJ. 1993;307(6896):125; BMJ. 1993;306(6890):1429-30; BMJ. 1993;307(6896):126-7; BMJ. 1993;397(6896):125-6.

15. Goldberg DP, Hillier VF. A scaled version of General Health Questionnaire. Psychol Med. 1979;9(1):139-45.

16. Codner MA, Wolfi JN, Anzarut A. Primary transcutaneous lower blepharoplasty with routine lateral canthal support: a comprehensive 10-year review. Plast Reconstr Surg. 2008; 121(1):241-50.

17. Barton FE Jr, Ha R, Awada M. Fat extrusion and septal reset in patients with the tear trough triad: a critical appraisal. Plast Reconstr Surg. 2004;113(7):2115-21; discussion 2122-3. Comment in: Plast Reconstr Surg. 2005;1 16(7):2035; Plast Reconstr Surg. 2005; 116(2):674-5; author reply 675

18. Dini GM, Quaresma MR, Ferreira LM. Translation into Portuguese, cultural adaptation and validation of the Rosenberg Self-Esteem Scale. Rev Soc Bras Cir Plast. 2004;19(1): 41-54.

19. Jelks GW, Glat PM, Jelks EB, Longaker MT. The inferior retinacular lateral canthoplasty: a new technique. Plast Reconst Surg. 1997;100(5):1262-70.

20. Lessa S, Sebastiá R, Flores E. Uma cantopexia simples. Rev Bras Oftalmol. 1999;58: 779-86.

21. Goldberg RA. The three periorbital hollows: a paradigm for periorbital rejuvenation (editorial). Plast Reconstr Surg. 2005;116(6):1796-804. Comment in: Plast Reconstr Surg. 2006;117(1):349

22. Pessa JE. An algorithm of facial aging: verification of Lambro's theory by three-dimensional stereolithography, with reference to the pathogenesis of midfacial aging, scleral show, and the lateral suborbital trough deformity. Plast Reconstr Surg. 2000; 106(2):479-88; discussion 489-90.

23. Hirmand H, Codner MA, McCord CD, Hester TR, Nahai F. Prominent eye: operative management in lower lid and midfacial rejuvenation and the morphologic classification system. Plast Reconstr Surg. 2002;110(2):620-8; discussion 629-34.

24. Patrocinio TG, Loredo BA, Arevalo CE, Patrocinio LG, Patrocinio JA. Complications in blepharoplasty: how to avoid and manage them. Braz J Otorhinolaryngol. 2011; 77(3):322-7.

25. Korn BS, Kikkawa DO, Cohen SR. Transcutaneous lower eyelid blepharoplasty with orbitomalar suspension: retrospective review of 212 consecutive cases. Plast Reconstr Surg. 2010;125(1):315-23

26. Parsa AA, Lye KD, Radcliffe N, Parsa FD. Lower blepharoplasty with capsulopapebral fascia hernia repair for palpebral bags: a long-term prospective study. Plast Reconstr Surg. 2008;121(4):1387-97. Comment in: Plast Reconstr Surg. 2008;122(6):1976-7; author reply 1977.

27. Ching S, Thoma A, McCabe RE, Antony MM. Measuring outcomes in aesthetic surgery: a comprehensive review of the literature. Plast Reconstr Surg. 2003;111(1):469-80; discussion 481-2. Comment in: Plast Reconstr Surg. 2003;112(7):1953-4; author reply 1954-5.

28. Figueroa C. Self-esteem and cosmetic surgery: is there a relation between the two? Plastic Surg Nurs. 2003:23(1):21-4. Review.

29. Kostanski M, Gullone E. Adolescent body image dissatisfaction: relationship with selfesteem, anxiety and depression controlling for body mass. J Child Psychol Psychiatry 1998:39(2):255-62. 